\title{
Estrés percibido y estrategias de afrontamiento en el equipo de enfermería: implicaciones para su formación
}

\author{
Rosario Isabel Niño-Higuera ${ }^{*}$, Xochitl Yadira Bravo-Paz', Maetzin Itzel Ordaz-Carrillo', \\ Carolina Santillan-Torres' y Mario Ricardo Gallardo-Contreras ${ }^{2}$ \\ 'Universidad Nacional Autónoma de México \\ ${ }^{2}$ Hospital Juárez de México
}

\begin{abstract}
Resumen: Los enfermeros atienden constantemente el dolor, la muerte, presiones laborales, pacientes problemáticos, etcétera; factores que favorecen el desarrollo de estrés laboral y tienen consecuencias de orden físico, social, emocional y conductual. Es importante identificar si existen perfiles diferentes de estrés laboral según los contextos y demandas específicas del lugar de trabajo. El objetivo fue evaluar el nivel de estrés percibido y su relación con variables sociodemográficas y las estrategias de afrontamiento predominantes en el personal de enfermería del servicio de Ortopedia. La muestra estuvo compuesta por 15 enfermeros, evaluados con la Escala de Estrés Percibido (EEP) de Cohen, Kamarak, y Mermelstein (1983) y el Inventario de Estrategias de Afrontamiento (IEA) de Lazarus y Folkman (1986). Los resultados mostraron un alto nivel de estrés percibido según el EEP, así como una alta percepción de desamparo y pérdida de control y una baja percepción de autoeficacia o control de las situaciones. No se encontraron diferencias significativas entre las Estrategias de Afrontamiento con los datos demográficos. Es esencial desarrollar intervenciones que faciliten la adquisición de habilidades de afrontamiento en el personal de enfermería que mejoren sus patrones de comportamiento y pensamiento e incrementen su nivel de autoeficacia.
\end{abstract}

Palabras clave: Estrés laboral, Estrategias de Afrontamiento, Enfermería, Estrés Percibido, Formación.

\section{Perceived stress and coping strategies in the nursing team: implications for their training}

\begin{abstract}
The nurses constantly attend to pain, death, work pressures, problematic patients, etcétera; factors that favor the development of work stress and have physical, social, emotional and behavioral consequences. It is important to identify if there are different profiles of work stress according to the contexts and specific demands of the workplace. The objective was to evaluate the level of perceived stress and its relationship with sociodemographic variables and coping skills predominant in the nursing staff of the Orthopedics service. The sample consisted of 15 nurses, evaluated with the Scale of Perceived Stress (SPS) of Cohen, Kamarak, and Mermelstein (1983) and the Inventory of Coping Skills (ICS) of Lazarus and Folkman (1986). The results showed a high level of perceived stress according to the SPS, as well as a high perception of helplessness and loss of control and a low perception of self-efficacy or control of situations. No significant differences were found in coping skills as a function of the demographic data. It is essential to develop interventions that facilitate the acquisition of coping skills in nurses who improve their behavior and thinking patterns and increase their level of self-efficacy.
\end{abstract}

Keywords: Work stress, Coping Skills, Nursing Staff, Perceived Stress, Training.

El estrés es uno de los factores psicológicos que más ha sido estudiado en los últimos años (Dobnik, Maletic, \& Skela-Savic, 2018; Gray-

Recibido: 28/09/2018 - Aceptado: 18/03/2019 - Avance online: 03/05/2019

*Correspondencia: Rosario Isabel Niño Higuera.

Universidad Nacional Autónoma de México.

C.P: 54743, México

E-mail: isabel.nino.higuera@gmail.com

Niño-Higuera, R.I., Bravo-Paz, X.Y., Ordaz-Carrillo, M.I., Santillan-Torres, C. y GallardoContreras, M.R. (Avance Online). Estrés percibido y estrategias de afrontamiento en el equipo de enfermería: implicaciones para su formación. Revista de Psicología Educación, 14(2), 157-166, https://doi.org/10.23923/rpye2019.02.180
Toft \& Anderson, 1981; Hinshaw \& Atwood, 1984; Martínez-López, \& López-Solache, 2005; Gómez, Puga, Mayán \& Gandoy, 2012; Quintanilla, 2004; Seleghim, Mombelli, Félix, Pagliarini \& Silva, 2012; Stordeur, D'hoore \& Vandenberghe, 2001). Sin embargo, es importante identificar que existen diferentes maneras de concebirlo. Selye (1978) menciona

1699-9517/@ 2019 Asociación Científica de Psicología y Educación (ACIPE). Publicado por Consejo General de Colegios Oficiales de Psicólogos, España. Este es un artículo Open Access bajo la CC BY-NC-ND licencia (http://creativecommons.org/licencias/bync-nd/4.0/. 
que el estrés es un patrón de reacciones fisiológicas y psicológicas que el individuo presenta de forma inespecífica a distintos estresores.

Lazarus y Folkman (1986) proponen el modelo transaccional del estrés en el que lo definen como una interacción entre el ambiente y el individuo, en donde la manera en la que el individuo percibe la demanda y los recursos que tiene para dar respuesta a la misma son fundamentales.

De igual manera, Lazarus y Folkman (1986) definen el afrontamiento como "aquellos esfuerzos cognitivos y conductuales que se desarrollan para manejar las demandas tanto internas como externas que son evaluadas como excedentes o desbordantes de los recursos del individuo" (p. 164).

Estos mismos autores clasifican el afrontamiento de dos formas diferentes: a) dirigida al problema, orientada a la modificación o cambio de la situación; y b) dirigida a la emoción, que implica reducir la respuesta emocional negativa a una situación estresante.

Zavala, Rivas, Andrade y Reidl (2008) mencionan que existen cinco estilos de afrontamiento presentes en la población mexicana (evasivo, distanciamiento, revaloración positivo, negación y análisis cognitivo-reflexivo); argumentan que en cada uno de los estilos de afrontamiento se encuentran diferencias en la percepcion que tiene el individuo de los estresores y la demanda.

El modelo de Karasek (1979) es una ampliación del modelo interactivo del estrés (Cox, 1978; Lazarus y Folkman, 1986) y explica que el estrés laboral se encuentra en relación proporcional a la amplitud de las demandas que se reciben del entorno y está modulado por la percepción de capacidad de autonomía o control que el sujeto considera que posee para realizar el trabajo (Moreno y Peñacoba, 1999); por tanto, la ausencia de percepción de control sobre las contingencias ambientales probabiliza la aparición del estrés.

El estrés es un fenómeno global y un término que se ha generalizado a hogares, familias y organizaciones. Dentro del ámbito laboral, afecta en mayor prevalencia a las profesiones que implican un contacto directo con las personas, principalmente en las que se destaca el cuidado a los otros (enfermeros, médicos, psicólogos), implica la combinación de diferentes variables tanto físicas, como psicológicas y sociales (Stordeur, D'hoore \& Vandenberghe, 2001; Gil-Monte, 2003).

Según la ONU (WHO, 2006), a nivel internacional hay una crisis global en ámbito de salud, ya que el personal de enfermería está disminuyendo su número debido a las altas demandas que incluye el servicio a los otros y los altos niveles de estrés laboral que implica. La exposición constante a atender el dolor, la muerte, las enfermedades terminales, las situaciones de urgencia, las demandas y presiones laborales, los conflictos de rol, los pacientes problemáticos, entre otros son algunos de los factores estresores más importantes de estrés laboral en el personal de enfermería (Molina \& Rodríguez, 2011 ; Orly, Rivka, Rivka \& Dorit, 2012; Khamisa, Peltzer, llic \& Oldenburg, 2017).

En el ámbito laboral, los estresores pueden ser definidos como las condiciones antecedentes entre el trabajo o la organización del mismo y las respuestas adaptativas por parte de los empleados (Jex \& Beehr, 1991). Gil-Monte (2003) menciona existen estresores como la escasez de personal, sobrecarga laboral, trabajo en turnos, pacientes problemáticos, falta de autonomía, contacto directo con la enfermedad, el dolor y la muerte, problemas con los niveles jerárquicos, falta de especificidad en las funciones, etcétera

Con relación a esto, Power y Sharp (1988) encontraron que la muerte, el dolor y la inadecuada preparación para manejar las demandas emocionales de familiares y pacientes son estresores más significativos que los conflictos con pares y el esfuerzo físico.

La exposición a los estresores anteriores provoca consecuencias de orden físico, social, emocional y conductual (Brunero, Cowan \& Fairbrother, 2008; Chang, Dally, Bidewell, Johnson, Lambert \& Lambert, 2006; Giraldo, López, Arango, Góez y Silva, 2013), así como respuestas psicofisiológicas como estrés mental, cambios de humor, burnout y, a su 
vez, afectan el desempeño y productividad del personal de enfermería (Molina \& Rodríguez, 2011 ; Orly, Rivka, Rivka \& Dorit, 2012).

Además de las consecuencias individuales del estrés laboral a nivel físico y familiar, éste también implica costos a nivel hospital u organizacional como el ausentismo, conflictos entre compañeros y rotación de personal (Maslach y Jackson, 1982). El estrés laboral también afecta el tipo de cuidados que se brindarán a pacientes y familiares (Firth, McKeown, Mclntee \& Britton, 1987; Maslach y Jackson, 1982).

Payne (2001) menciona que es posible que algunos factores contribuyan al desarrollo y mantenimiento del estrés y otros factores que promueven protección (estrategias de afrontamiento).

Chang et al. (2006) y Lambert, Lambert, Petrini, Li y Zhang (2007) indican que el personal de enfermería utiliza estrategias de afrontamiento centradas en la emoción para mejorar su salud mental como autocontrol, reevaluación positiva, evitación funcional y distanciamiento. Ceslowitz (1989) encontró que las enfermeras que experimentan niveles menores de burnout son las que emplean métodos de solución de problemas planificados.

Se han diseñado diferentes intervenciones para abordar el estrés laboral; sin embargo, una de las intervenciones más efectivas es la intervención cognitivo conductual, que se centran en brindar estrategias para reducir las respuestas a nivel fisiológico con ejercicios de relajación como la respiración, relajación autógena y relajación muscular progresiva; y en los elementos cognitivos y conductuales haciendo énfasis en la necesidad de información sobre la función y los elementos del estrés, la búsqueda de nuevas interpretaciones de un mismo evento, la adquisición de habilidades de solución de problemas y el desarrollo de habilidad de comunicación asertivas (Baeyer \& Krause, 1983; Brunero, Cowan \& Fairbrother, 2008; Orly, Rivka, Rivka, \& Dorit, 2012).

Duquette, Kérowc, Sandhu, y Beaudet (1994) identifica tres grupos de factores de protección para el estrés laboral: factores organizacionales como la distribución de tareas; factores de afrontamiento; y factores demográficos como la edad, el grado profesional y los años de experiencia.

Por tanto, la respuesta a los estresores será afectada directamente por las diferencias individuales de los trabajadores asociadas a los factores sociodemográficos y el tipo de estrategias que utilizan (Duquette et al. 1994). Con base en esto, es importante identificar si existen perfiles diferentes de estrés laboral según los distintos contextos y las demandas específicas del servicio en el que se encuentren.

Servellen y Leake (1993) mencionan que variables sociodemográficas como la edad, el estado civil, años de experiencia, turno del trabajo, etcétera, no parecen influir en el nivel de estrés.

Sin embargo, hay investigaciones que argumentan que existe una edad de vulnerabilidad para la presencia de estrés laboral que está relacionada a los primeros años de la carrera profesional (Applebaum, 1981; Cherniss 1980; Edelwich y Brodsky, 1980; como se citó en Moreno y Peñacoba, 1999).

Cherniss, (1980, como se citó en Moreno y Peñacoba, 1990) indica que el estrés laboral no puede separarse del sistema de respuesta y afrontamiento del propio individuo, resaltando la importancia que tienen las variables individuales en la génesis y desarrollo del burnout.

Por tanto, el objetivo general del presente estudio fue evaluar el nivel de estrés percibido en el personal de enfermería del servicio de Ortopedia de un hospital de especialidades de la Ciudad de México.

Como objetivos específicos se planteó analizar la existencia de diferencias significativas basadas en variables sociodemográficas y las estrategias de afrontamiento predominantes en el personal de enfermería.

\section{MÉTODO}

\section{PARTICIPANTES}

El estudio se llevó a cabo en un hospital de especialidades de la Ciudad de México. La muestra estuvo compuesta por quince participantes de enfermería del servicio de 
Ortopedia del turno matutino, seleccionadas mediante un muestreo no probabilístico intencional. De los participantes 14 fueron mujeres y 1 hombre, con una media de edad de 41.47 años con un rango de edad de 26 a 50 años y una desviación estándar de \pm 7.415. Cinco casados, tres solteros, tres separados, tres viviendo en unión libre y uno viudo.

Siete con carrera técnica, seis con licenciatura y dos con posgrado. Ocho con más de 20 años de experiencia laboral; cinco con menos de diez años y dos de entre 10 a 20 años de experiencia laboral. Doce enfermeras generales, dos jefes de enfermería y un auxiliar de enfermería.

\section{INSTRUMENTOS DE MEDIDA}

Escala de Estrés Percibido de Cohen, Kamarak, y Mermelstein (1983) adaptación mexicana de González y Landero (2007): compuesto por 14 preguntas en escala tipo Likert con cinco opciones de respuesta, que van de 0 (nunca) a 4 (muy frecuentemente). Está dividido en dos factores: Factor 1. Percepción de desamparo o pérdida de control; Factor 2. Autoeficacia percibida o control de estímulos estresantes. La puntuación mínima posible es de 0 y la máxima de 56 , siendo las puntuaciones más altas correspondientes a un mayor estrés percibido. El instrumento cuenta con una adecuada consistencia interna $(\alpha=$ .83), con el Factor 1 explicando un $42.8 \%$ de la varianza y el Factor 2, un 53.2\%, y una consistencia interna individual de $\alpha=.83$ y $\alpha$ $=.78$, respectivamente.

Inventario de Estrategias de Afrontamiento de Lazarus y Folkman (1988) adaptación mexicana de Zavala, Rivas, Andrade y Reidl (2008): compuesto por 20 preguntas en escala tipo Likert con cuatro opciones de respuesta: $0=\mathrm{No}_{\mathrm{o}}$ de ninguna manera, $1=$ En alguna medida, $2=$ Generalmente y 3 =Siempre, pudiéndose obtener una puntuación total mínima de 0 y máxima de 60. El alfa de Cronbach total del instrumento es .85. El instrumento se encuentra dividido en cinco factores por cada una de las estrategias de afrontamiento: 1. Enfrentamiento evasivo $(\alpha=.75), 2$. Enfrentamiento de distanciamiento $(\alpha=.74), 3$. Enfrentamiento reevaluación positiva $(\alpha=.68), 4$. Enfrentamiento análisis cognitivo-reflexivo $(\alpha=.68)$ y 5 . Enfrentamiento de negación $(\alpha=.64)$.

\section{PROCEDIMIENTO}

Fase 1 - Revisión Bibliográfica. Se realizó una revisión de la literatura relacionada con estrés laboral en el personal de enfermería, así como los instrumentos utilizados para medir el nivel de estrés percibido y las estrategias de afrontamiento utilizadas por el personal de salud. Se decidió utilizar los instrumentos adaptados para población mexicana la Escala de Estrés Percibido (González y Landero, 2007) y el Inventario de Estrategias de Afrontamiento (Zavala et al., 2008). Posteriormente, se diseñó la propuesta de investigación y se presentó en una cita con la Jefe de Enfermería del servicio de Ortopedia para explicarle el objetivo y el procedimiento a seguir.

Fase 2 - Aplicación de los instrumentos. Una vez autorizado el proyecto, se inició con la evaluación del personal de enfermería, las evaluaciones se llevaron a cabo dentro de los horarios laborales de los participantes (8:00 am. a 14:00 pm.) para mayor comodidad. Cada uno de los instrumentos fue contestado de manera voluntaria, bajo la firma de un consentimiento informado y bajo confidencialidad. La muestra fue intencional, no probabilística, lo que facilitó en gran medida la aplicación de los instrumentos. La evaluación de la muestra tardó aproximadamente una semana en llevarse a cabo debido a que los participantes respondían los cuestionarios en el horario laboral y lo intercalaban con diferentes actividades.

Fase 3 - Análisis de la Información. Una vez aplicados los instrumentos, los datos se concentraron en una base de datos en el software estadístico SPSS 21.0 en donde se compararon los niveles de estrés percibido y sus subescalas (percepción de desamparo y autoeficacia percibida) con las variables 
demográficas (edad, estado civil, escolaridad, años de experiencia laboral y jerarquía), para calcular si las diferencias podrían considerarse como significativas se utilizó la prueba no paramétrica de Kruskall Wallis para muestras independientes. Además, se calculó el tamaño del efecto de dichas diferencias mediante el programa G*Power. Finalmente, se compararon las estrategias de afrontamiento predominantes en el personal de enfermería del servicio de Ortopedia.

Fase 4 - Devolución de la información. Para finalizar, se reunió al personal de enfermería evaluado y se les expusieron los resultados encontrados con el apoyo de una presentación en Power Point con una breve explicación del modelo transaccional del estrés, compuesta por definición de estrés, definición de estrategias de afrontamiento y los resultados encontrados.

\section{RESULTADOS}

La Figura 1 muestra el puntaje obtenido en la Escala de Estrés Percibido, se observa que el puntaje promedio de nivel de estrés percibido total es de 23, 27 lo que indica un nivel alto de estrés en el personal de enfermería y una desviación típica de \pm 7.516. De igual manera, en ambos factores se muestra un nivel alto, en percepción de desamparo una media de 14.93 y una desviación típica de \pm 5.189; mientras que en autoeficacia percibida, encontramos una media de 8.33 y una desviación típica de \pm 3.244 .

Por otro lado, la Figura 2. nos muestra el puntaje obtenido en el Inventario de Estrategias de Afrontamiento, en el que se encontró que la estrategia más utilizada por el personal de enfermería fue el distanciamiento con una media de 9.2 y una desviación típica de 2.704; seguidas por reevaluación positiva
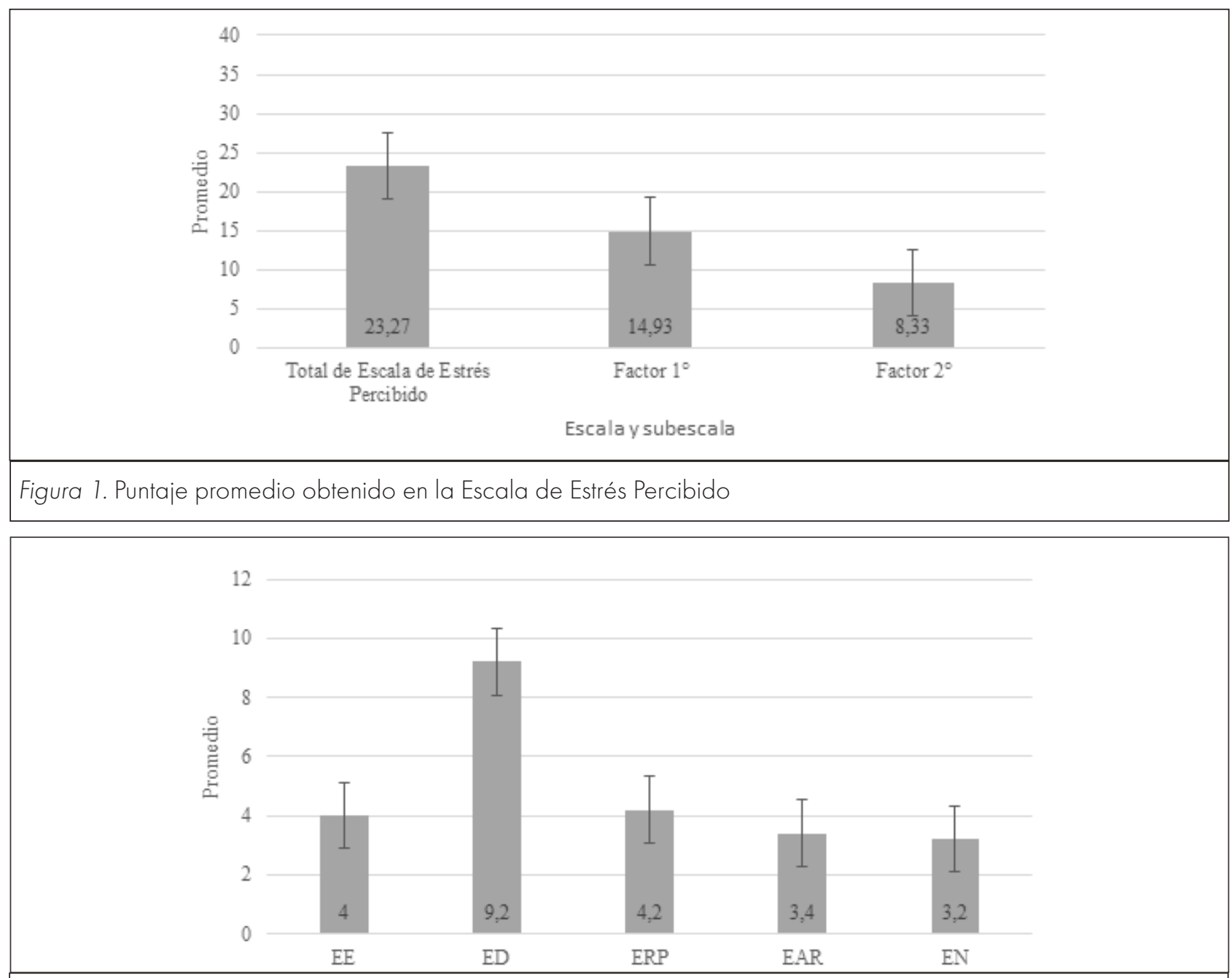

Figura 2. Puntaje promedio obtenido en el Inventario de Estrategias de Afrontamiento. 
Tabla 1

Total de Puntaje Promedio obtenido en la Escala de Estrés Percibido según rangos de edad

\begin{tabular}{|c|c|c|c|c|}
\hline $\begin{array}{l}\text { Rangos } \\
\text { de Edad }\end{array}$ & $\begin{array}{l}\text { Número } \\
\text { de } \\
\text { Casos }\end{array}$ & $\begin{array}{l}\text { Total de } \\
\text { Estrés } \\
\text { Percibido }\end{array}$ & $\begin{array}{c}\text { Factor } \\
1^{a}\end{array}$ & $\begin{array}{c}\text { Factor } \\
2^{a}\end{array}$ \\
\hline $\begin{array}{l}20 \text { a } 29 \\
\text { años }\end{array}$ & 2 & 15.50 & 9.50 & 6.00 \\
\hline $\begin{array}{c}30 \text { a } 39 \\
\text { años }\end{array}$ & 2 & 22.50 & 14.00 & 8.50 \\
\hline $\begin{array}{c}\text { De } 40 \\
\text { años en } \\
\text { adelante }\end{array}$ & 12 & 24.82 & 16.09 & 8.73 \\
\hline \multicolumn{5}{|c|}{$\begin{array}{l}\text { Nota: } \\
\text { Factor } 1^{a} \text { : Percepción de desamparo o pérdida de control } \\
\text { Factor } 2^{a} \text { : Autoeficacia percibida o control de estímulos } \\
\text { estresantes. }\end{array}$} \\
\hline
\end{tabular}

con una media de 4.2 y una desviación típica de +2.823 y la evasión de las circunstancias con una media de 4 y una desviación típica de +2.330 .

En la Tabla 1 se muestra el total de puntaje promedio obtenido según los rangos de edad, se observa que el nivel de estrés percibido es alto en personas mayores a los 30 años, así como la percepción de desamparo y en coincidencia con el nivel de autoeficacia percibida. Sin embargo, las diferencias entre los tres grupos de edad no pueden ser consideradas como significativas partiendo de las pruebas estadísticas utilizadas $\left(x^{2}=2.91, g l=2, p=.233\right)$, observándose un tamaño del efecto bajo $(d=0.26)$.

En la Tabla 2 se muestra el total de puntaje promedio obtenido según la jerarquía, se observa que el nivel de estrés percibido es alto en cualquier nivel de la jerarquía (auxiliar de enfermería, enfermero general o jefe), siendo más elevado en los enfermeros generales y coincidiendo de igual manera con el nivel de percepción de desamparo y la autoeficacia percibida. Al ser alto en los distintos niveles de jerarquía no es posible observar diferencias significativas entre ellos $\left(x^{2}=.826, g l=2\right.$, $p=.662)$, teniendo un tamaño igualmente bajo $(d=.1437)$.

En la Tabla 3 se muestra el total de puntaje promedio obtenido según el nivel de estudios, se observa que el nivel de estrés percibido
Tabla 2

Total del Puntaje Promedio obtenido en la Escala de Estrés Percibido según la Jerarquía

\begin{tabular}{|c|c|c|c|c|}
\hline Jerarquía & $\begin{array}{l}\text { Número } \\
\text { de } \\
\text { Casos }\end{array}$ & $\begin{array}{l}\text { Total de } \\
\text { Estrés } \\
\text { Percibido }\end{array}$ & $\begin{array}{c}\text { Factor } \\
1^{a}\end{array}$ & $\begin{array}{c}\text { Factor } \\
2^{a}\end{array}$ \\
\hline $\begin{array}{l}\text { Auxiliar de } \\
\text { Enfermería }\end{array}$ & 1 & 20.00 & 13.00 & 7.00 \\
\hline $\begin{array}{l}\text { Enfermero } \\
\text { (a) } \\
\text { General }\end{array}$ & 12 & 24.17 & 15.25 & 8.92 \\
\hline Jefe & 2 & 19.50 & 14.00 & 5.50 \\
\hline \multicolumn{5}{|c|}{$\begin{array}{l}\text { Nota: } \\
\text { Factor } 1^{a}: \text { Percepción de desamparo o pérdida de control } \\
\text { Factor } 2^{a} \text { : Autoeficacia percibida o control de estímulos } \\
\text { estresantes. }\end{array}$} \\
\hline
\end{tabular}

es elevado sin importar nivel de estudios, siendo más elevado en aquellos con niveles de posgrado y coincidiendo de igual manera con el nivel de percepción de desamparo y la autoeficacia percibida. A pesar de que el nivel de estrés se encuentra más alto en la población con mayor grado de estudios, es indispensable resaltar que dichas diferencias no se pueden considerar como significativas $\left(x^{2}=.826, g\right)=2$, $p=.662)$ aunque con un tamaño del efecto mediano $(d=.30)$.

\section{Tabla 3}

Total del Puntaje Promedio obtenido en la Escala de Estrés Percibido según el nivel de estudios

\begin{tabular}{|c|c|c|c|c|}
\hline $\begin{array}{l}\text { Nivel de } \\
\text { estudios }\end{array}$ & $\begin{array}{l}\text { Número } \\
\text { de } \\
\text { Casos }\end{array}$ & $\begin{array}{l}\text { Total de } \\
\text { Estrés } \\
\text { Percibido }\end{array}$ & $\begin{array}{c}\text { Factor } \\
1^{a}\end{array}$ & $\begin{array}{c}\text { Factor } \\
2^{a}\end{array}$ \\
\hline $\begin{array}{l}\text { Carrera } \\
\text { Técnica }\end{array}$ & 7 & 26.29 & 16.86 & 9.43 \\
\hline Licenciatura & 6 & 18.67 & 12.00 & 6.67 \\
\hline Posgrado & 2 & 26.50 & 17.00 & 9.50 \\
\hline \multicolumn{5}{|c|}{$\begin{array}{l}\text { Nota: } \\
\text { Factor } 1^{a} \text { : Percepción de desamparo o pérdida de control } \\
\text { Factor } 2^{a} \text { : Autoeficacia percibida o control de estímulos } \\
\text { estresantes. }\end{array}$} \\
\hline
\end{tabular}


situacionales según el modelo interactivo del estrés propuesto por Lazarus y Folkman (1986), las características personales juegan un papel en el desarrollo de ésta, si bien no como una variable independiente si como una variable moduladora de los efectos principales.

Una de las consecuencias del estrés es la pérdida de satisfacción laboral que repercute en la calidad de las tareas realizadas, la calidad laboral y la productividad a nivel organizacional y el impacto a nivel individual del personal de salud y sus familiares, caracterizado por repercusiones en la salud, problemas familiares, dificultad de concentración, entre otros (Duquette et al., 1994; Michalik, Zawadka, Wolski, Stanisławek, \& Węgorowski, 2017).

Por tanto, como bien lo demuestran Said y Khen (2018) es importante desarrollar intervenciones que permitan la adquisición de habilidades de afrontamiento ante el estrés laboral que mejoren los patrones de comportamiento y pensamiento incrementando el nivel de autoeficacia y favoreciendo un afrontamiento efectivo a las situaciones de estrés; y al mismo tiempo permitan estandarizar protocolos de enseñanza.

Resulta esencial brindar estrategias que favorezcan el afrontamiento funcional a las demandas ambientales y situacionales del estrés laboral, así como herramientas individuales que faciliten la adaptación y propicien la percepción de control en el personal de enfermería.

Como mencionan Said y Khen (2018) es importante favorecer la implementación de programas preventivos que permitan al personal de enfermería desarrollar habilidades para hacer frente al ámbito hospitalario y laboral.

Considerando los contextos hospitalarios como contextos formativos, es esencial focalizar la atención a estudiantes y practicantes quienes, de igual manera, se encuentran expuestos a los estímulos ambientales y sociales que favorecer el desarrollo de estrés, de percepción de desamparo y disminución de la autoeficacia; por lo que, el desarrollo de estos programas preventivos debe tener como objetivo brindar herramientas y estrategias a implementar en estos contextos y fortalecer las estrategias que permitan reducir y/o eliminar los efectos a nivel individual, social y laboral del estrés percibido.

Entre las limitaciones del presenta trabajo se identifica que la aplicación de los instrumentos se realizó dentro del entorno laboral de los participantes lo que sesga en cierta medida la confiabilidad de los mismos acorde con las demandas del ambiente (exigencias de los jefes, carga de trabajo, entre otros). El personal evaluado no constituía el total de la población del personal de enfermería del servicio de Ortopedia lo que limita los resultados y dificulta la probabilidad de generalización de los resultados. Utilizar otros instrumentos para complementar los niveles obtenidos, ya que existen otros instrumentos con estas mismas características diseñados específicamente para medir las variables de interés del presente estudio. Otra de las limitaciones y quizá la más importante es la falta de distribución de la muestra, la población no era equilibrada debido a la misma condición del muestreo limitando el análisis al uso de pruebas no paramétricas, así como la probabilidad de realizar un análisis del tamaño del efecto más robusto.

\section{- Conflicto de intereses.}

Los autores declaran no tener ningún conflicto de intereses.

\section{REFERENCIAS}

Brunero, S., Cowan, D., \& Fairbrother, G. (2008). Reducing emotional distress in nurses using cognitive behavioral therapy: A preliminary program evaluation. Japan Journal of Nursing Science, 5(2), 109-1 15. doi: $10.1111 /$ i.1742-7924.2008.00102.x

Castillo, I., Torres, N., Ahumada, A., Cárdenas, K., y Licona, S. (2014). Estrés laboral en enfermería y factores asociados. Cartagena (Colombia). Salud Uninorte, 30 (1), 34-43.

Ceslowitz, S. (1989). Burnout and coping strategies, among hospital staff nursing. Journal of Advanced Nursing, 14, 553-558. doi: https://doi. org/10.1111/i.1365-2648.1989.tb01590.x 
Chang, E., Dally, J., Hancock, K., Bidewell, J., Johnson, A., Lambert, V., \& Lambert, C. (2006). The relationships among workplace stressors, coping methods, demographic characteristics and health in Australian nurses. Journal of Professional Nursing, 22(1), 30 38. doi: 10.1016/i.profnurs.2005.12.002

Cohen, S., Kamarck, T., \& Mermelstein, R. (1983). A Global Measure of Perceived Stress. Journal of Health and Social Behavior, 24(4), 385. doi: 10.2307/2136404

Cox, T. (1978). Stress. Londres: McMillan.

De Souza, J., Costa, P., Hoffmeiste, E., Souza, B., Pinheiro, B. y Poli, C., (201 1). Workplace stress in nursing workers from an emergency hospital: Job Stress Scale analysis. Revista LatinoAmericana de Enfermagem, 19(5),112231. doi: http://dx.doi.org/10.1590/S010411692011000500009

Dobnik, M., Maletic, M. \& Skela-Savic, B. (2018). Work-Related stress factors in nurses at Slovenian hospitals - A cross-sectional study. Slovenian Journal of Public Health, 57(4), 192-200. doi: 10.2478/siph-2018$\underline{0024}$

Duquette, A., Kérowc, S., Sandhu, B. K., \& Beaudet, L. (1994). Factors Related to Nursing Burnout A Review of Empirical Knowledge. Issues in Mental Health Nursing, 15(4), 337-358. doi: http://dx.doi. org/10.3109/01612849409006913

Firth, H., McKeown, P., Mclntee, J., \& Britton, P. (1987). Professional depression, "burnout" and personality in longstay nursing. International Journal of Nursing Studies, 24(3), 227-237. doi: http://dx.doi. org/10.1016/0020-7489(87)90005-8

Gil-Monte, P. (2003). El síndrome de quemarse en el trabajo en profesionales de enfermería. Revista da Facultad da Educacao da UFC, INTER-ACAO, 1(1), 19-33.

Giraldo, Y., López, B., Arango, L., Góez, F. y Silva, E. (2013). Estrés laboral en auxiliares de enfermería, Medellín-Colombia, 2011. Revista de la Universidad Industrial de Santander. Salud, 45(1), 23-31.

Gómez, C., Puga, A., Mayán, J. y Gandoy, M. (2012). Niveles de estrés en el personal de enfermería de unidades de cuidados paliativos. GEKOMOMOS, 23(2), 59-62.

González, M., \& Landero, R. (2007). Factor Structure of The Perceived Stress Scale (PSS) in a Sample from Mexico. The Spanish Journal of Psychology, 10, 199-206. doi: https://doi. org/10.1017/S1138741600006466

Gray-Toft, P., \& Anderson, J. G. (1981). Stress among hospital nursing staff: Its causes and effects. Social Science \& Medicine. Part A: Medical Psychology \& Medical Sociology, 15(5), 639-647. doi: https://doi. org/10.1016/0271-7123(81)90087-0

Hinshaw, A. S., \& Atwood, J. R. (1984). Nursing staff turnover, stress, and satisfaction: Models, measures, and management. In: Werley H. \& Fitzpatrick J. (eds). Annual review of nursing research (pp. 133-153). Springer, Berlin, Heidelberg. DOI: https://doi. org/10.1007/978-3-662-40453-9_6

Jex, S. M., \& Beehr, T. A. (1991). Emerging theoretical and methodological issues in the study of work-related stress. Research in Personnel and Human Resources Management, 9, 311-365.

Karasek, R. A. (1979). Job Demands, Job Decision Latitude, and Mental Strain: Implications for Job Redesign. Administrative Science Quarterly, 24(2), 285. dio: http:// dx.doi.org/10.2307/2392498

Khamisa, N., Peltzer, K., Ilic, D., \& Oldenburg, B. (2017). Effect of personal and work stress on burnout, job satisfaction and general health of hospital nurses in South Africa. Health SA Gesondheid, 22, 252 - 258. DOI: https:// doi.org/10.1016/i.hsag.2016.10.001

Lambert, V. A., Lambert, C. E., Petrini, M., Li, X. M., \& Zhang, Y. J. (2007). Predictors of physical and mental health in hospital nurses within the People's Republic of China. International Nursing Review, 54(1), 85-91. doi: $10.1111 /$ i.1466-7657.2007.00512.x

Lazarus, S. y Folkman, R.S. (1986). Estrés y procesos cognitivos. Barcelona: Martínez Roca.

Maslach, C. y Jackson, S.E. (1982). Burnout in health professions: A Psychological Analysis. In G. Sanders y J. Suls, (Eds.). Social 
Psychology of Health and Illness. Hillsdale, N.J.: Lawrence Herlbaum.

Martínez-López, C. y López-Solache, G. (2005). Características del síndrome de Burnout en un grupo de enfermeras mexicanas. Archivos en Medicina Familiar. Vol. 7(1). 6-9.

Michalik, J., Zawadka, M., Wolski, D., Stanisławek, A. \& Węgorowski, P. (2017). The professional stress of nurses employed in medical institutions in the Lublin Region. Journal of Education, Health and Sport, 7(8), 296-308. doi:10.5281/zenodo.847992

Molina, M.J. y Rodríguez, N. (2011). Estrategias de afrontamiento: un programa de entrenamiento para paramédicos de la Cruz Roja. Revista Costarricense de Psicología, 30, 17-33.

Moreno y Peñacoba (1999). El estrés asistencial en los servicios de salud. En. Manual de psicología de la salud: fundamentos, metodología y aplicaciones. M. A. Simón (coord.). Madrid: Biblioteca Nueva. 739757.

Orly, S., Rivka, B., Rivka, E., \& Dorit, S.-E. (2012). Are cognitive-behavioral interventions effective in reducing occupational stress among nurses? Applied Nursing Research, 25(3), 152-157. doi: 10.1016/i. apnr.2011.01.004.

Payne, N. (2001). Occupational stressors and coping as determinants of burnout in female hospice nurses. Journal of Advanced Nursing, 33(3), 396-405. doi: https://doi. org/10.1046/i.1365-2648.2001.01677.x

Power, K. G., \& Sharp, G. R. (1988). A comparison of sources of nursing stress and job satisfaction among mental handicap and hospice nursing staff. Journal of Advanced Nursing, 13(6), 726-732. doi: https://doi. org/10.1111/i.1365-2648.1988.tb00563.x

Quintanilla, M. (2004). Prevalencia del síndrome de Burnout en las enfermeras de la Unidad de Paciente Crítico del Hospital del Trabajador Santiago de Chile y una propuesta de intervención. Revista Chilena de Medicina Intensiva, 19(1), 33-38.
Said, Z. \& Khen, G. (2018). A review on mindfulness and nursing stress among nurses. The International Journal of Counseling and Education, 3(1), 1-13. doi: $10.23916 / 0020170211740$

Seleghim, M., Mombelli, M., Félix, M., Pagliarini, M. y Silva, S. (2012). Sintomas de estresse em trabalhadoras de enfermagem de uma unidade de pronto socorro. Revista Gaúcha de Enfermagem, 33(3), 165-173. doi: https://dx.doi.org/10.1590/S198314472012000300022

Selye, H. (1978). The Stress of Life. México: McGraw-Hill.

Servellen, G. V. y Leake, B. (1993). Burnout in Hospital nurses: a comparison of acquired inmunodeficiency síndrome, oncology, general medical and intensive care unit nurses examples. Journal of Professional Nursing. 9, 169-177. doi: https://doi. org/10.1016/8755-7223(93)90069-O

Stordeur, S., D'Hoore, W., \& Vandenberghe, C. (2001). Leadership, organizational stress, and emotional exhaustion among hospital nursing staff. Journal of Advanced Nursing, 35(4), 533-542. doi: https://doi.org/10.1046/ j.1365-2648.2001.01885.x

Von Baeyer, C., \& Krause, L. (1984). Effectiveness of Stress Management Training for Nurses Working in a Burn Treatment Unit. The International Journal of Psychiatry in Medicine, 13(2), 113-126. doi: https://doi. org/10.2190/WPV9-OY55-55TX-FMX1

World Health Organization (2006). New global alliance seeks to address worldwide shortage of doctos, nurses and other health workers. Recuperado de: http://www.who.int/ mediacentre/news/releases/2006/pr26/en/

Zavala, L., Rivas, R., Andrade, P. y Reidl, L. (2008). Validación del instrumento de estilos de enfrentamiento de Lazarus y Folkman en adultos de la Ciudad de México. Revista Intercontinental de Psicología y Educación, 10(2), 159-182. 